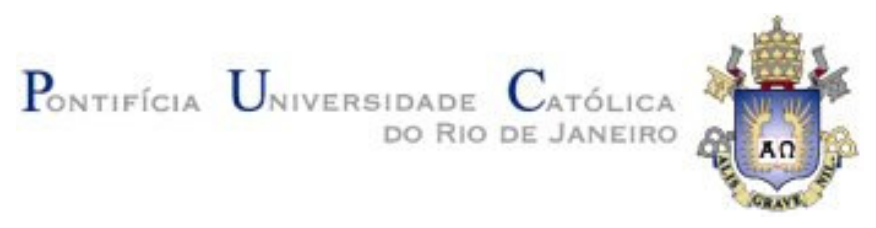

Elder José Reioli Cirilo

\title{
GenArch: Uma Ferramenta Baseada em Modelos para Derivação de Produtos de Software
}

\section{Dissertação de Mestrado}

Dissertação apresentada como requisito parcial para obtenção do grau de Mestre pelo Programa de Pósgraduação em Informática do Departamento de Informática do Centro Técnico Científico da PUC-Rio.

Orientadores: Carlos José Pereira de Lucena Uirá Kulesza 

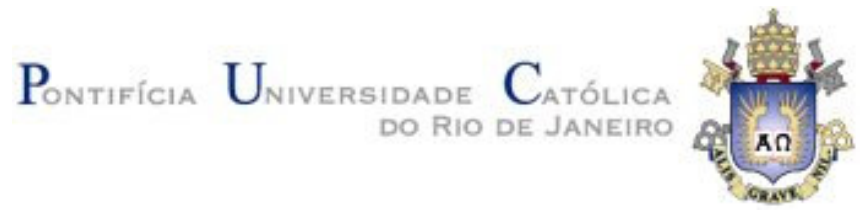

Elder José Reioli Cirilo

\title{
GenArch: Uma Ferramenta Baseada em Modelos para Derivação de Produtos de Software
}

\begin{abstract}
Dissertação apresentada como requisito parcial para obtenção do grau de Mestre pelo Programa de Pósgraduação em Informática do Departamento de Informática do Centro Técnico Científico da PUC-Rio. Aprovada pela Comissão Examinadora abaixo assinada.
\end{abstract}

Prof. Carlos José Pereira de Lucena

Orientador

Departamento de Informática - PUC-Rio

Prof. Uirá Kulesza

Co-Orientador

Departamento de Informática e Matemática Aplicada - UFRN

Prof. Arndt von Staa

Departamento de Informática - PUC-Rio

Prof. Ivan Mathias Filho

Departamento de Informática - PUC-Rio

Prof. José Eugenio Leal

Coordenador Setorial do Centro

Técnico Científico 
Todos os direitos reservados. É proibida a reprodução total ou parcial do trabalho sem autorização da universidade, do autor e do orientador.

Elder José Reioli Cirilo

Graduou-se no Curso de Bacharelado em Ciência da Computação da Universidade Federal de Juiz de Fora (UFJF) em 2006.

Ficha Catalográfica

\begin{abstract}
Cirilo, Elder José Reioli
GenArch: Uma ferramenta baseada em modelos para derivação de produtos de software / Elder José Reioli Cirilo ; orientador: Carlos José Pereira de Lucena ; coorientador: Uirá Kulesza. - 2008.
\end{abstract}

100 f. : il. ; $30 \mathrm{~cm}$

Dissertação (Mestrado em Informática)-Pontifícia Universidade Católica do Rio de Janeiro, Rio de Janeiro, 2008.

Inclui bibliografia

1. Informática - Teses. 2. Linhas de produtos de software. 3. Desenvolvimento Dirigido por Modelos. 4. Engenharia de Domínio. 5. Ferramentas para Derivação de Linha de Produtos de Software. I. Lucena, Carlos José Pereira de. II. Kulesza, Uirá. III. Pontifícia Universidade Católica do Rio de Janeiro. Departamento de Informática. IV. Título.

CDD: 004 


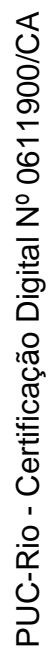

A Deus e aos meus pais José Cyrilo e Maria Josefina 


\section{Agradecimentos}

Me sinto privilegiado por ter conhecido Uirá e ganhado a oportunidade de tê-lo como um dos meus orientadores. Esse trabalho não teria sido possível sem suas idéias, seus ensinamentos e principalmente, sua amizade. Serei sempre grato a ele por tudo isso.

Me sinto especialmente honrado por ter professor Lucena como um dos meus orientadores. Gostaria de agradecê-lo pelo acolhimento, liberdade, confiança e ensinamentos. Sei que tudo isso tornou a realização desse trabalho mais simples.

Agradeço a todos os professores do Departamento de Informática que contribuíram indiretamente para realização desse trabalho. Em especial gostaria de agradecer aos Professores Arndt von Staa e Renato Cerqueira que contribuíram para enriquecer meus conhecimentos. Gostaria de agradecer também a todos os colegas da PUC-Rio que tive o prazer de conquistar durante meu mestrado: Andrew, Carol, Camila, Cidiane, Cláudio (Baiano), Dani, Ingrid, Maíra, Márcio, Pádua e Rodrigo. Em especial, gostaria de agradecer a Paulo e Sergio que me acompanharam desde os meus primeiros dias de PUC-Rio e a Roberta minha primeira companheira de pesquisa que muito me ensinou e contribuiu para minha formação.

Um agradecimento especial a todos os amigos de Juiz de Fora, em especial, Carolina, Daniel Barral, Daniel Nicácio, Edmar, Fábio, Júlio, Poliana, Rafael, Vinícios, Tiago Melquiades, Thiago Senador.

Agradeço a PUC-Rio e a CAPES pelo apoio financeiro necessário a realização desse trabalho.

Gostaria de agradecer também as pessoas mais importantes da minha vida, minha família. Aos meus pais, José Cyrilo e Maria Josefina, pelo amor, carinho e confiança que sempre depositaram em mim. A minha tia Maria da Conceição por ser uma segunda mãe. As minha irmãs, Elaina e Elisana, e as minhas sobrinhas Maria Rita e Manuela pelos sorrisos e pensamento positivo. A Flávia, minha namorada, que sempre me manteve animado nos momentos difíceis da realização desse trabalho.

Finalmente agradeço a Deus, o realizador de todas essas maravilhas em minha vida. 


\section{Resumo}

Cirilo, Elder José Reioli; Lucena, Carlos José Pereira de; Kulesza, Uirá GenArch: Uma Ferramenta Baseada em Modelos para Derivação de Produtos de Software. Rio de Janeiro, 2008. 100p. Dissertação de Mestrado - Departamento de Informática, Pontifícia Universidade Católica do Rio de Janeiro.

Este trabalho apresenta uma ferramenta baseada em modelos para derivação de produtos de LPSs, denominada GenArch. O objetivo principal da ferramenta é permitir que a comunidade de desenvolvimento de software tradicional, utilize conceitos e fundamentos de abordagens de LPSs na produção de seus sistemas ou partes de seus sistemas sem a necessidade do entendimento de modelos e conceitos complexos. A abordagem implementada pela ferramenta foi elaborada com base em fundamentos do desenvolvimento dirigido por modelos. Centrada na definição de três modelos (características, implementação e configuração), a ferramenta permite a derivação automática de produtos ou frameworks existentes. O trabalho também define um conjunto específico de anotações Java que possibilitam a geração automática dos modelos de derivação a partir dos elementos de implementação da arquitetura de uma LPS. A plataforma Eclipse e as tecnologia EMF e openArchitectureWare foram utilizadas como base para a implementação da ferramenta. Uma extensão da ferramenta que atende especificamente aos modelos de componente Spring e OSGi, também é proposta nessa dissertação. Tal extensão permite a instanciação automática da LPS e aplicações através de diferentes tipos de customizações, variando da configuração fina de propriedades de componentes até a seleção automática de quais componentes irão compor o produto final. Como parte de validação da abordagem, a ferramenta foi utilizada na derivação automática de três diferentes estudos de caso: (i) o framework JUnit; (ii) uma LPS de jogos J2ME; e (iii) uma aplicação web baseada em serviços. Diversas lições aprendidas e resultados do uso da ferramenta nestes três diferentes cenários são também apresentadas.

\section{Palavras-chave}

Linhas de Produtos de Software; Desenvolvimento Generativo; Desenvolvimento Dirigido por Modelos, Engenharia de Domínio, Ferramentas para Derivação de Linha de Produtos de Software 


\section{Abstract}

Cirilo, Elder José Reioli; Lucena, Carlos José Pereira de; Kulesza, Uirá GenArch: A Model-Based Product Derivation Tool. Rio de Janeiro, 2008. 100p. Msc Dissertation - Departamento de Informática, Pontifícia Universidade Católica do Rio de Janeiro.

This work presents a model-based tool for product derivation, called GenArch, which aims to enable the mainstream software developer community to use the concepts and foundations of the SPL approach, without the need to understand complex concepts or models. The tool approach is build on top of model-driven development techniques. It is centered on the definition of three models (feature, implementation and configuration models), which enable the automatic instantiation of software product lines (SPLs) or frameworks. A set of specific Java annotations are also defined to allow generating automatically many of the models, based on existing implementations elements of SPL architectures. The Eclipse platform, and EMF and openArchitectureWare technologies are used as the base for the implementation of the tool. The dissertation also presents a GenArch extension that addresses the new abstractions provided by the Spring and OSGi component models. Different kinds of customizations are provided by this extension varying from fine-grained configuration of component properties to the automatic selection of components that will compose the final product. As part of the approach validation, the tool was used in the derivation of three case studies: (i) JUnit framework; (ii) a J2ME games SPL; (iii) a service oriented Web application. Several lessons learned and discussions resulting from the use of the tool also are described.

\section{Keywords}

Software Product Lines; Generative Programming; Model-Driven Development, Domain Engineering, Software Product Lines Derivation Tool 


\section{Sumário}

1 Introdução 14

1.1. Problemas $\quad 15$

1.2. Limitação das Abordagens Atuais 16

1.3. Solução Proposta 16

$\begin{array}{ll}\text { 1.4. Objetivos } & 17\end{array}$

1.5. Organização do Texto 17

2 Desenvolvimento de Linhas de Produtos de Softwares 19

2.1. Linhas de Produtos de Software 19

2.2. Desenvolvimento Generativo 23

2.3. Implementando Linhas de Produtos de Software 25

2.3.1. Frameworks Orientados a Objetos 25

2.3.2. Orientação a Aspectos 26

2.3.3. Tecnologia de Componentes $\quad 27$

2.4. Ferramentas para Derivação de Produtos de Software Baseadas em $\begin{array}{ll}\text { Modelo de Características } & 29\end{array}$

$\begin{array}{ll}\text { 2.4.1. Gears } & 29\end{array}$

2.4.2. pure::variants 31

2.5. Conclusão 33

3 GenArch: Uma Ferramenta Baseada em Modelos para Derivação de

Produtos de Software $\quad 35$

3.1. Visão Geral da Abordagem $\quad 35$

3.1.1. Anotação do código-fonte com características e variabilidades 36

3.1.2. Modelos de Derivação 37

3.1.3. Criação Automática da Estrutura de Templates 38

3.1.4. Refinamento e Sincronização dos Modelos de Derivação 39

3.1.5. Documentação de Variabilidades 40

3.1.6. Processo de Derivação de Produtos 41

3.2. Arquitetura e Implementação da Ferramenta GenArch 41

3.2.1. Visão Geral da Arquitetura 42 
3.2.2. Eclipse Modeling Framework (EMF) 44

3.2.3. openArchitectureWare (oAW) 47

3.2.3.1. Estendendo as funcionalidades da linguagem XPand 48

3.2.4. Feature Modeling Plug-in 49

3.3. Conclusão 50

4 Estudos de Caso

4.1. Framework JUnit 52

4.1.1. Anotando código do JUnit com Características e Variabilidades 54

4.1.2. Gerando versões iniciais dos modelos 55

4.1.3. Implementando Variabilidades do JUnit com Templates 58

4.1.4. Instanciando o JUnit 60

4.2. Linha de Produtos para Jogos J2ME 62

4.2.1. Descrição do Estudo de Caso 62

4.2.2. Anotando Características na LPS para Jogos J2ME 64

4.2.3. Gerando os Modelos do jogo Rain of Fire 64

Derivando Jogos para Diferentes Modelos de Celular 67

$\begin{array}{ll}\text { 4.2.4. } & 67\end{array}$

$\begin{array}{ll}\text { 4.3. Sumário } & 68\end{array}$

5 Derivando Aplicações Baseadas em Spring e OSGi 69

5.1. Spring e OSGi - Tecnologias de Componentes 69

5.2. Integração das Tecnologias Spring e OSGi 71

5.3. Arquitetura da Extensão 73

5.3.1. Estendendo os modelos GenArch para suportar Spring e OSGi 74

5.3.2. Construção automática dos modelos a partir de artefatos Spring e OSGi 75

5.3.3. Derivando produtos Spring/OSGi 80

5.4. Conclusões e Resultados 83

6 Trabalhos Relacionados $\quad 85$

6.1. Abordagem para Derivação de Linhas de Produtos de Softaware 85

6.2. Abordagem para Instanciação de Framewoks $O \bigcirc \quad 86$

6.3. Abordagem para Configuração Automática de Aplicações Corporativas 88

7 Conclusão 90

$\begin{array}{ll}\text { 7.1. Contribuições } & 91\end{array}$ 
7.2. Discussões e Lições Aprendidas

92

7.3. Trabalhos Futuros

94

7.4. Uso da Ferramenta GenArch na Indústria

Referências 


\section{Lista de Figuras}

Figura 1. Modelo de Domínio Generativo 23

Figura 2. Elementos da ferramenta Gears $\quad 30$

Figura 3. Processo de derivação do pure::variants 33

Figura 4. Visão geral da abordagem 36

Figura 5. Exemplo de template criado automáticame 39

Figura 6. Arquitetura da ferramenta GenArch 43

Figura 7. Meta-modelo do modelo de implementação 45

Figura 8. Meta-modelo do modelo de configuração 45

Figura 9. Meta-modelo do modelo de derivação 46

Figura 10. Fluxo de execução do plug-in oAW (Stahl et al. 2006) 48

Figura 11. Modelo de característica do Framework JUnit 50

Figura 12. Arquitetura Orientada a Aspectos do JUnit 53

Figura 13. Classe TestCase anotada $\quad 54$

Figura 14. Versão Inicial dos Modelos GenArch do JUnit 55

Figura 15. Aspect RepeatedTestGeneric anotado 56

Figura 16. Modelos GenArch do JUnit - Versão Final 57

Figura 17. Mapeamento entre um elemento de implementação e uma $\begin{array}{ll}\text { característica } & 58\end{array}$

$\begin{array}{ll}\text { Figura 18. TestCaseTemplate } & 59\end{array}$

Figura 19. Configuração do modelo de característica 60

$\begin{array}{ll}\text { Figura 20. RepeatedTestGenericTemplate } & 61\end{array}$

Figura 21. Janela de derivação $\quad 62$

Figura 22. Aspecto LoadlmgOnDemand e LoadlmgOnInit com anotações GenArch 64

Figura 23. Modelos GenArch do jogo Rain of Fire 66

Figura 24. Configurações do modelo de característica do jogo Rain of Fire 67

Figura 25. Jogo Rain of Fire executando em modelos diferentes de celulares 68

Figura 26. Arquitetura Spring/OSGi da aplicação JPetStore 72

$\begin{array}{ll}\text { Figura 27. Classe Java anotada } & 76\end{array}$

Figura 28. Versão inicial dos modelos de implementação e deployment da $\begin{array}{ll}\text { aplicação JPetStore } & 78\end{array}$

Figura 29. Versão final dos modelos de derivação da aplicação JPetStore $\quad 79$ 
Figura 30. Versão inicial dos modelos de configuração e características 80

Figura 31. Versão final dos modelos de configuração e características 80

Figura 32. Template de um arquivo de configuração Spring 82 


\section{Lista de Tabelas}

Tabela 1. Anotações GenArch e seus atributos $\quad 37$

Tabela 2. Pacotes gerados automaticamente pelo EMF 46

Tabela 3. Funções de extensão 48

Tabela 4. Anotações GenArch para Spring/OSGi e seus atributos 76 